\title{
SOME NUMERICAL RESULTS OF MULTIPOINTS BOMNDARY VALUE PROBLEMS ARISE
} IN ENVIRONMENTAL PROTECTION

\author{
Daniel N. Pop \\ Assistant professor, Faculty of Engineering, Department of Computers and Electrical Engineering"Lucian \\ Blaga" University of Sibiu, Sibiu, Romania, nicolae.pop@ulbsibiu.ro
}

Abstract: In this paper, we investigate two problems arise in pollutant transport in rivers, and we give some numerical results to approximate this solutions. We determined the approximate solutions using two numerical methods: 1. B-splines combined with Runge-Kutta methods, 2. BVP4C solver of MATLAB and then we compare the run-times.

Key words: B-spline, Nonlocal and multipoint boundary value problem, Multistep, Runge-Kutta and extrapolation method. AMS Classifications (2010): 65D07,34B10,65L06

\section{The statement of the problems}

The aim of this work is to compare the run-times of two numerical methods used to determine the approximate solutions of multipoints value problems with boundary conditions at infinity, they appears in pollutants transport in rivers.

Ames and Lohner (1981), [1], study models for a transport, reaction and dissipation of pollutants in rivers. One model gives rise to a system of three first-order PDE in one space variable $\mathrm{x}$ and time t. By looking for traveling wave solutions, that depend only the variable: $\mathrm{z}=\mathrm{x}-\mathrm{t}$

they reduce PDE, to ODE:

$$
\begin{gathered}
\mathrm{z}=\mathrm{x}-\mathrm{t} \text { they reduce } \mathrm{PDE}, \text { to ODE like: } \\
\mathrm{f}^{\prime \prime}=\beta \mathrm{gf} \\
\mathrm{g}^{\prime \prime}=-\beta \mathrm{gh} \\
\mathrm{h}^{\prime \prime}=\lambda \beta \mathrm{gh}
\end{gathered}
$$

Here $\mathrm{f}$ represents a pollutant, $\mathrm{g}$ bacteria, and $\mathrm{h}$ carbon; the physical parameters $\lambda$ and $\beta$ are constants. After showing that the equations for $\mathrm{g}$ and $\mathrm{h}$ imply that:

$$
\mathrm{g}(\mathrm{z})=\mathrm{E}-(\mathrm{h}(\mathrm{z}) / \lambda)
$$

where $\mathrm{E}$ is a given value for $\mathrm{g}(\infty)$. They reduce the system of ODE so:

$$
\begin{aligned}
& \mathrm{h}^{\prime \prime}=\lambda \beta(\mathrm{E}-(\mathrm{h} / \lambda)) \mathrm{h}, \\
& \mathrm{f}^{\prime \prime}=\beta(\mathrm{E}-(\mathrm{h} / \lambda)) \mathrm{f} .
\end{aligned}
$$

This equations are be solved subject to boundary conditions:

$$
h(0)=1, h(\infty)=0, f(0)=1, f(\infty)=0
$$

Since $h(\infty)=0$ the equation (3) can be approximated for large $\mathrm{z}$ by:

$$
\mathrm{h}^{\prime \prime}=\mathrm{E} \lambda \beta \mathrm{h}
$$

For $\beta=\lambda=10$ and $E=1$, this equation becomes:

with solution:

$$
\mathrm{h}^{\prime \prime}=100 \mathrm{~h}
$$

$$
\mathrm{h}(\mathrm{z})=\mathrm{Ae} 10 \mathrm{z}+\mathrm{Be}-10 \mathrm{z}
$$

Because $\mathrm{h}(0)=1, \mathrm{~h}(\infty)=0$ implies $\mathrm{A}=0$ and $\mathrm{B}=1$, then the approximate solution $\mathrm{h}(\mathrm{z})$ of the problem $(6)$ with conditions (5) is asymptotically multiple of e-10z 
With $h(z)=e-10 z, \beta=\lambda=10, E=1$ the equation (4) becomes:

$$
\mathrm{f}^{\prime \prime}=10(1-\mathrm{e}-10 \mathrm{z} / 10) \mathrm{f} \text {, }
$$

subject to boundary conditions:

$$
f(0)=1, f(\infty)=0
$$

Also for large $\mathrm{z}$ the equation (7) can be approximate by:

$$
\mathrm{f}^{\prime \prime}=10 \mathrm{f}
$$

Solving this approximating ODE we find that:

$$
f(z)=A \text { e } \sqrt{ } 10 z+B e-\sqrt{ } 10 z
$$

For constants $A, B$. The solution is to have $f(\infty)=0$, so $A=0$.

From this we see that the solution of the given equation is approximately a multiply e- $\sqrt{10 z}$. For practical purposes it is interesting solution behavior in transient area (an interval bounded by that starts from 0 ) and not the behavior of the asymptotically area where $\mathrm{h}(\mathrm{z}) \simeq 0, \mathrm{f}(\mathrm{z}) \simeq 0$.

\section{Numerical results}

We present two approximation methods for exact solutions of the problems (6) and (7) with conditions (5), and (8). We divide the interval $[0, \infty)$ in two subintervals like:

1. a transient area $[0,10]$ and

2. the asymptotically area $(10, \infty)$,

where $\mathrm{z}=10$ is the positive solution of equation:

\subsection{B-splines and Runge-Kutta methods}

$$
\mathrm{r}^{2}-100=0 \text {. }
$$

A natural choice of the start solution for this problem is:

since:

$$
h(t)=1 /(t+1)^{3}, f(t)=1 /(t+1)^{3}
$$

$$
\begin{aligned}
& h(0)=1, h(\infty)=0, \\
& f(0)=1, f(\infty)=0 .
\end{aligned}
$$

We obtained the approximation solutions of the problem (6) with conditions (5), like:

1. On the interval $[0,10]$ with $B$-splines functions of order $(k+1)$,

2. And on $[10, \delta$ ) with Runge-Kutta methods (k-stages), solver ode113 or ode45 ([4]).

The convergence given in ([3], [5]).

After 5 iterations obtained the following results fig :1(a), fig:1(b):

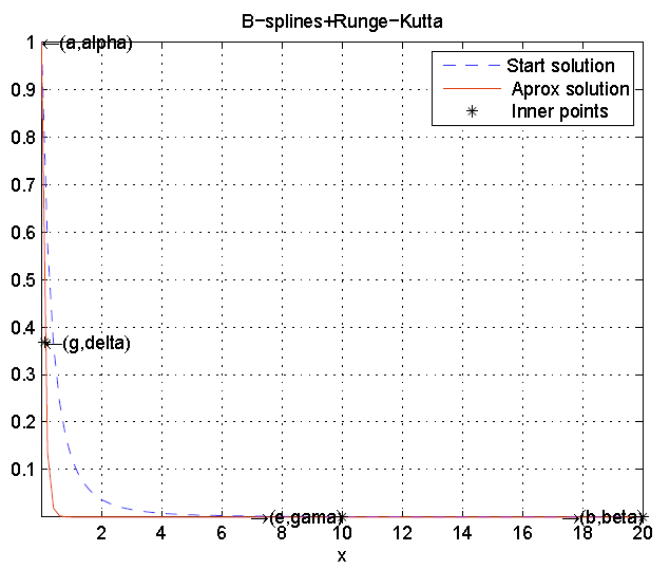

(a) Approximate solution for the problem: (6)+ (5)

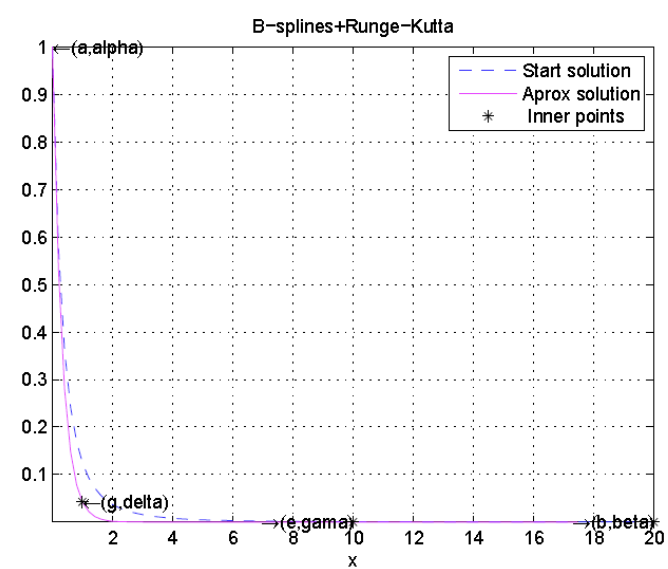

(b) Approximate solution for the problem: (7)+ (8)

Figure 1: B-spline + Runge-Kutta

\subsection{The solver BVP4C}

Following the idea giving in [2, pp:146-152], we solve the problems $(6)+(5)$ and (7) $+(8)$ using the solver BVP4c of MATLAB suggest by [6, pp: 153] and obtained the following results: 


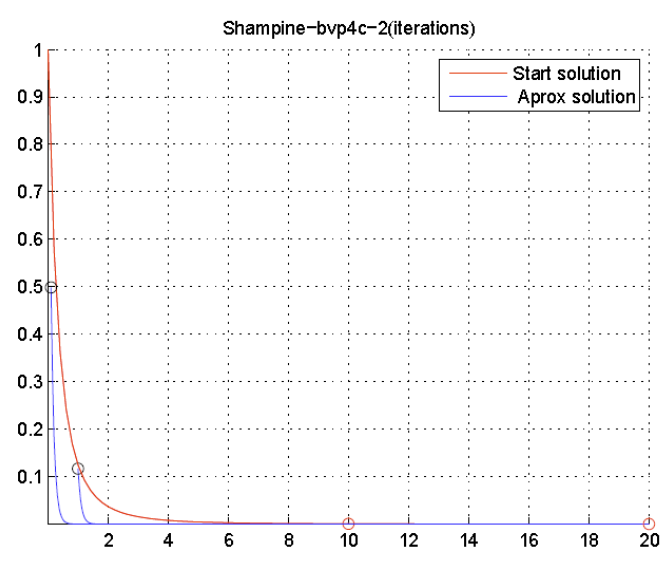

(a) Approximate solution for the problem: (6)+ (5)

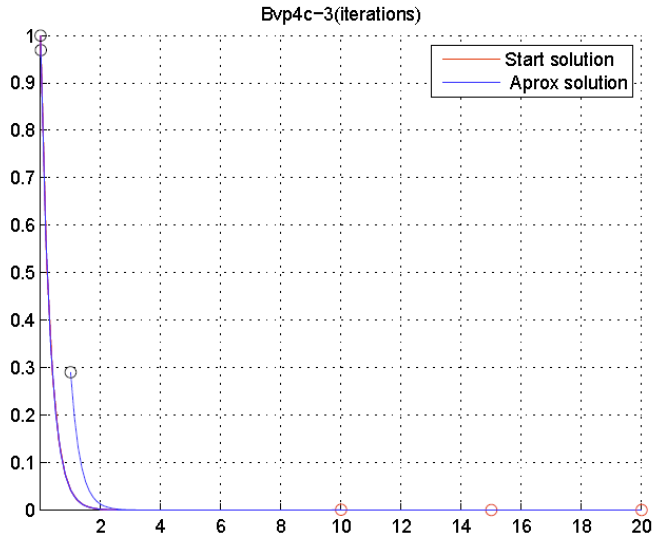

(b)Approximate solution for the problem: (7)+ (8)

Figure 2: BVP4C

\section{Conclusions}

Using the functions tic-toc of Malab we obtained the following results:

\begin{tabular}{|l|l|l|l|}
\hline Problems & Method & Tolerance & Running Times \\
\hline$(6)+(5)$ & B-Splines+Runge-Kutta & $10^{-15}$ & 1.645805 seconds \\
\hline$(6)+(5)$ & BVP4C & $10^{-15}$ & 2.1725 seconds \\
\hline$(7)+(8)$ & B-Splines+Runge-Kutta & $10^{-15}$ & 0.931149 seconds \\
\hline$(7)+(8)$ & BVP4C & $10^{-15}$ & 2.0004 seconds \\
\hline
\end{tabular}

For more details concerning the run-times you can be used the function profile viewer of Matlab. This problem is set on an infinite interval, so some experimentation is necessary to verify that a sufficiently large $\delta$ has been specified.

\section{References}

- [1] W.Ames, E.Lohner, Nonlinear models of reaction-diffusssion in rivers, New

- Brunswick, NJ:IMACS, 1981.

- [2] U.M. Ascher, R.M. Mattheij, R.D Russel, Numerical Solution of boundary Value Problems for Ordinary Differential Equations Philadelphia S.I.A.M, 1995.

- [3] G. Goldner, Radu T.Tr^ımbita ,s, A combined method for two point boundary value problem, P.U.M.A, Vol 11 pag 255-264, 2000.

- [4] Matlab 2011, The MathWorks. Inc.,Natick MA, USA.

- [5] Daniel N. Pop, Radu T.Tr^ımbita, s, An approximation methods for second order nonlinear value polylocal problems using B-splines and Runge-Kutta methods, N.A.T 2010, Studia Univ "Babes-Bolyai" Cluj-Napoca, Vol. LVI, Number 2 , pag 515-526, June 2011.

- [6] L.F. Shampine, I.Gladwell, S.Thomson, Solving ODEs with Matlab, Cam- bridge University Press,2003. 